\section{Neuropsychological Deficit Scale}

Molly E. Zimmerman

Department of Psychology, Fordham University, Bronx, NY, USA

\section{Definition}

The neuropsychological deficit scale was developed by Reitan and Wolfson $(1988,1993)$ as a summary measure of an individual's overall neuropsychological functioning.

\section{Current Knowledge}

The neuropsychological deficit scale, also known as the general neuropsychological deficit scale, is derived from 42 variables from the HalsteadReitan neuropsychological test battery (Reitan and Wolfson 1993). The 42 variables comprise 19 scores based on level of performance across a range of neuropsychological functions, 9 motor and sensory-perceptual scores, 2 scores shown to differentiate between individuals with and without brain damage, and 12 dysphagia and constructional dyspraxia scores. Each of the 42 variables is scored from a range of 0 ("a perfectly normal performance") to 3 ("a severely impaired performance"). The total scale interpretation is as follows: normal (0-25), mild impairment (26-40), moderate impairment (41-67), and severe impairment (68-168). More information on scale development and scoring can be found in Reitan and Wolfson (1993), and data on validation can be found in Wolfson and Reitan (1995).

\section{Cross-References}

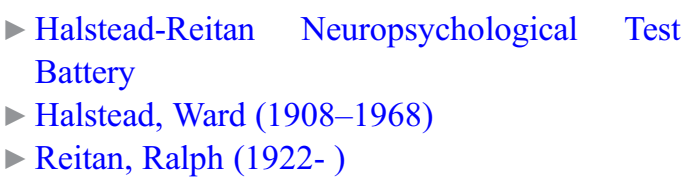

\section{References and Readings}

Reitan, R. M., \& Wolfson, D. (1988). Traumatic brain injury (Vol. II). Tuscon: Neuropsychology Press.

Reitan, R. M., \& Wolfson, D. (1993). The Halstead-Reitan neuropsychology test battery: Theory and clinical interpretation (2nd ed.). Tuscon: Neuropsychology Press.

Wolfson, D., \& Reitan, R. M. (1995). Cross-validation of the general neuropsychological deficit scale (GNDS). Archives of Clinical Neuropsychology, 10(2), 125-131. 